\title{
PREDATOR-PREY AND HOST-PARASITE SPATIAL STOCHASTIC MODELS ${ }^{1}$
}

\author{
By RinAldo B. ScHINAZI \\ University of Colorado
}

\begin{abstract}
We consider two interacting particle systems on $\mathbf{Z}^{d}$ to model predatorprey and host-parasite interactions. In both models we have two types of particles (1 and 2) and each site in $\mathbf{Z}^{d}$ can be in one of four states: empty, occupied by a type 1 particle, occupied by a type 2 particle or occupied by two particles (one of each type). Each type gives birth to particles of the same type on nearest neighbor sites. The interaction between the two types of particles occurs only when a site is occupied by one particle of each type. For both models we show that coexistence and noncoexistence are possible in any dimension.
\end{abstract}

1. The models. We start with a predator-prey model. Each site in $\mathbf{Z}^{d}$ can be in one of four states: empty, occupied by a 1, occupied by a 2 , or occupied by a 1 and a 2 . This last state will be denoted by $(1,2)$. We think of 1 as being the prey and 2 as being the predator. Prey and predators give birth (at different rates) on nearest neighbor sites. Predators on a site with no prey die at a certain fixed rate. When a site is occupied by a predator and prey [state $(1,2)$ ] then the predator eats the prey at a fixed rate and only the predator remains on the site. This is the only interaction between the two species.

We now give a mathematical description of the model. Consider a continuous time Markov process $\eta_{t}$ on $\{0,1,2,(1,2)\}^{\mathbf{Z}^{d}}$. If the process is in state $\eta$, we say that site $x$ is empty, occupied by a prey, occupied by a predator or occupied by a prey and a predator according to whether $\eta(x)$ equals $0,1,2$ or $(1,2)$, respectively. The evolution of the process depends on four parameters $\lambda_{1}>0, \lambda_{2}>0, \phi>0$ and $\delta_{2}>0$. Denote by $\|\cdot\|$ the Euclidean norm and for $x \in Z^{d}, \eta \in\{0,1,2,(1,2)\}^{\mathbf{Z}^{d}}$ let

$$
\begin{aligned}
& n_{1}(x, \eta)=\operatorname{card}\left(\left\{y \in Z^{d}:\|y-x\|=1 \text { and } \eta(y)=1 \text { or } \eta(y)=(1,2)\right\}\right) \\
& n_{2}(x, \eta)=\operatorname{card}\left(\left\{y \in Z^{d}:\|y-x\|=1 \text { and } \eta(y)=2 \text { or } \eta(y)=(1,2)\right\}\right) .
\end{aligned}
$$

Received March 1996; revised July 1996.

${ }^{1}$ Partially supported by NSF Grant DMS-96-25126.

AMS 1991 subject classification. 60K35.

Key words and phrases. Predator-prey, host-parasite, interacting particle systems. 
A site $x$ changes its state in the configuration $\eta$ according to the following transition rates:

$$
\begin{aligned}
& 0 \rightarrow 1 \text { at rate } \lambda_{1} n_{1}(x, \eta) \\
& 2 \rightarrow(1,2) \text { at rate } \lambda_{1} n_{1}(x, \eta) \\
& 0 \rightarrow 2 \text { at rate } \lambda_{2} n_{2}(x, \eta) \\
& 1 \rightarrow(1,2) \text { at rate } \lambda_{2} n_{2}(x, \eta) \\
& (1,2) \rightarrow 2 \text { at rate } \phi \\
& 2 \rightarrow 0 \text { at rate } \delta_{2} .
\end{aligned}
$$

In words, we allow creation of prey at rate $\lambda_{1} n_{1}$ on empty sites or sites occupied by predators. We allow creation of predators at rate $\lambda_{2} n_{2}$ on empty sites or sites occupied by prey. A predator on a site that is not occupied by a prey dies at rate $\delta_{2}$. Finally, a prey is eaten by a predator at rate $\phi$ if they are at the same site. Observe that there is at most one prey and one predator per site.

In order to formulate our results, we need to introduce the contact process $\xi_{t}$ on $\{0,1\}^{\mathbf{Z}^{d}}$. The evolution of the contact process is governed by the following rules. The transition rates for a site $x$ in a configuration $\xi$ are

$$
\begin{aligned}
& 0 \rightarrow 1 \text { at rate } \lambda n_{1}(x, \xi), \\
& 1 \rightarrow 0 \text { at rate } \delta,
\end{aligned}
$$

where $\lambda$ and $\delta$ are positive parameters. Note that $\delta_{0}$ (the measure that concentrates on the all 0's configuration) is a stationary distribution for the contact process. There is also a stationary distribution $\nu$ called the upper invariant distribution of the contact process. For every initial distribution $\xi_{0}$ in $\{0,1\}^{Z^{d}}$, there is a $c \geq 0$ such that

$$
\xi_{t} \text { converges in distribution to } c \delta_{0}+(1-c) \nu \text {. }
$$

There is a critical value $\lambda_{c}(\delta)$ such that if $\lambda \leq \lambda_{c}(\delta)$ then $\nu=\delta_{0}$ and $\xi_{t}$ converges to $\delta_{0}$. If $\lambda>\lambda_{c}(\delta)$ then $\nu \neq \delta_{0}$ and $\nu$ concentrates on configurations with infinitely many 1's. See Bezuidenhout and Grimmett (1990) for these results; see also Liggett (1985) and Durrett (1988) for general results about the contact process.

We now go back to our predator-prey model.

THEOREM 1. Consider the predator-prey model on $\mathbf{Z}^{d}$ for $d \geq 1$. For any $\lambda_{1}>0, \lambda_{2}>0$ and $\delta_{2} \geq 0$ there is $f\left(\lambda_{1}, \lambda_{2}\right)$ such that if $\phi<f$ then predators and prey may coexist. More precisely, there is a stationary distribution that concentrates on configurations with infinitely many sites occupied by $(1,2)$.

Observe that if we start the process without prey (1's) then the predators (2's) evolve exactly like a contact process. If $\lambda_{2}$ and $\delta_{2}$ are such that $\lambda_{2}>\lambda_{c}\left(\delta_{2}\right)$, then the 2's will survive even in the absence of 1's but Theorem 1 tells us that if $\phi$ is small enough the 2's do not take over the whole space and 
coexistence is possible. On the other hand, if we make the biologically meaningful hypothesis that predators die out in the absence of prey, that is, if we pick $\lambda_{2}$ and $\delta_{2}$ such that

$$
\lambda_{2}<\lambda_{c}\left(\delta_{2}\right),
$$

then Theorem 1 tells us that for $\phi$ small the 2's survive in the presence of 1's (the 2's would die out in the absence of 1's).

Theorem 1 is proved using a renormalization technique invented by Bramson and Durrett (1988) [see also Durrett (1995)]. The idea of the proof is quite simple: if $\phi=0$ then the 1's evolve independently of the 2's and a site $(1,2)$ never changes its state, so 1's and 2's survive. By a continuity argument this is still true for small $\phi>0$. However, some care must be exercised in the application of the technique here since this process is not monotone in any useful way.

If $\delta_{2}=\infty$, it means that a 2 at a site with no 1 dies immediately. So each site may be in either one of three states: 0,1 or $(1,2)$. This is exactly the model considered by Schinazi (1996) for an epidemic except that here the state $(1,2)$ plays the role of the state 2 there. Theorem 1 holds in this case as well ( $f$ does not depend on $\delta_{2}$ ) and it proves coexistence for a different range of the parameters than what was previously known: Schinazi (1996) proved that if $\lambda_{2}>\lambda_{c}(\phi)$ and if $\lambda_{1}$ is large enough then there is coexistence of 1's and 2's when $\delta_{2}=\infty$ (see Theorem 1 there).

If $\lambda_{2}<\lambda_{c}\left(\delta_{2}\right)$, it is intuitively clear that the predators should not deplete the prey since predators would die out if there were no prey. This is confirmed by the following theorem.

THEOREM 2. For all $\lambda_{2}$ and $\delta_{2}$ such that $\lambda_{2}<\lambda_{c}\left(\delta_{2}\right)$ and all $\lambda_{1}$ there is $F\left(\lambda_{2}, \delta_{2}\right)>0$ such that if $\phi>F$, then for any initial configuration the predators die out. That is, for any finite subset of $Z^{d}(d \geq 1)$ there is almost surely a finite time after which no site in the subset will ever be occupied by $a$ 2.

Theorem 2 is proved using a renormalization argument as in Durrett and Schinazi (1993) [see also Schinazi (1996)]. This time we first consider the system with $\phi=\infty$. This means that each time a 1 and a 2 are in the same site, the 1 disappears immediately. It is easy to see that in this situation the 1's have no role in the evolution of the 2's. The 2's are a subcritical contact process that dies out exponentially fast. By a continuity argument we prove that the 2's also die out for $\phi$ large enough.

In view of Theorems 1 and 2 , it is natural to ask if $f=F$. In other words, for fixed $\lambda_{1}, \lambda_{2}$ and $\delta_{2}$, is there $\phi_{c}$ such that if $\phi<\phi_{c}$, there is coexistence, while if $\phi>\phi_{c}$, the 2's die out? We conjecture that there is such a $\phi_{c}$ but we have no proof of this. The difficulty comes form the fact that we do not know whether the process is monotone in $\phi$.

We now turn to the other system we are interested in: The host-parasite model. We think of 1 as being a host and 2 as being a parasite. Here too we 
have four possible states for each site of $\mathbf{Z}^{d}$ : 0 (empty), 1, 2 and $(1,2)$. The evolution is identical to the predator-prey model except for one transition:

$$
(1,2) \rightarrow 0 \text { at rate } \phi \text {. }
$$

That is, we assume that if the parasite kills the host it will also die. We also assume that the 2's do not survive in the absence of 1's:

$$
\lambda_{2}<\lambda_{c}\left(\delta_{2}\right) \text {. }
$$

This model was inspired by the (nonspatial) model considered by Isham (1995) for which the interaction between the host and the parasite is only through the additional mortality that the parasite induces.

Theorem 1 holds for the host-parasite model as well and the proof is the same as for the predator-prey model. This is so because the two models are identical when $\phi=0$.

Theorem 2 also holds for the host-parasite model with $F=\delta_{2}$. For if $\phi>\delta_{2}$, then a 2 dies at rate $\delta_{2}$ while a $(1,2)$ dies at rate $\phi>\delta_{2}$. So the 2's are dominated by a contact process with death rate $\delta_{2}$ and birth rate $\lambda_{2}$. Since $\lambda_{2}<\lambda_{c}\left(\delta_{2}\right)$ the 2's die out.

Theorems 1 and 2 agree with the observation that a parasite may persist in a population if and only the additional mortality that a parasite induces is not too large.

When $\phi>\delta_{2}$ as explained above, the 2's in the host-parasite model are dominated by a subcritical contact process. So the 2's die out exponentially fast and this implies that the 1's take over the whole space. For a proof of a very similar result, see Theorem 3 in Schinazi (1996). For the predator-prey model such a result should be true for $\lambda_{2}<\lambda_{c}\left(\delta_{2}\right)$ and for $\phi$ large enough, but we have no proof except for $\phi=\infty$. In this case there are no sites $(1,2)$ and the 2's evolve exactly like a contact process with birth rate $\lambda_{2}$ and death rate $\delta_{2}$. So the 2's die out exponentially fast and the 1's take over.

Coexistence results are usually not easy to prove; see for instance Durrett (1993, 1995), Durrett and Neuhauser (1991) and Kang, Krone and Neuhauser (1995). The type of model we consider here is usually more difficult to analyze because it is not monotone in any apparent way. However, allowing sites to be occupied by the two types of particles at the same time greatly simplifies the problem and we are able to give short proofs for both coexistence and noncoexistence. We feel that allowing both types at the same site does not diminish the biological relevance of our models. There are many models in the mathematical biology literature for which proofs of coexistence are not known [see, for instance, Sato, Matsuda and Sasaki (1994) and Andjel and Schinazi (1996)]. We feel that modifications of the models in the spirit of this paper may lead to more tractable problems.

2. Proof of Theorem 1. The predator-prey and the host-parasite models may be constructed with Harris' graphical representation [Harris (1972)] that uses independent sequences of Poisson processes. For a construction of the same type of model, see Schinazi (1996). 
We will show coexistence for the predator-prey model on $\mathbf{Z}$ and then explain why our proof implies coexistence on $\mathbf{Z}^{d}$ for $d \geq 2$. We will compare the predator-prey model to a simple oriented percolation model. In order to do so we first need some notation. Let

$$
\begin{gathered}
\mathscr{L}=\left\{(m, n) \in \mathbf{Z}^{2}: m+n \text { is even }\right\}, \\
B=(-4 L, 4 L) \times[0, T], \quad B_{m, n}=(2 m L, n T)+B, \\
I=[-L, L], \quad I_{m}=2 m L+I,
\end{gathered}
$$

where $L$ and $T$ are parameters to be chosen later. We also consider the boundaries of the boxes:

$$
\begin{aligned}
C & =([-4 L, 4 L] \times\{0\}) \cup(\{-4 L\} \times[0, T]) \cup(\{4 L\} \times[0, T]), \\
C_{m, n} & =C+(2 m L, n T) .
\end{aligned}
$$

We declare $(m, n) \in \mathscr{L}$ wet if the process, starting with every site in $I_{m}$ occupied by $(1,2)$ at time $n T$, is such that every site of $I_{m-1}$ and every site of $I_{m+1}$ has a $(1,2)$ at time $(n+1) T$. Moreover we want the above event to happen regardless of the states of the sites on the boundary $C_{m, n}$ of $B_{m, n}$. This last condition is important in order to relate what happens in the finite box $B_{m, n}$ with what happens for the infinite system since we do not have any monotonicity in this process. Observe that the events $\{(m, n)$ is wet $\}$ and $\{(j, k)$ is wet $\}$ are independent if $(j, k)$ and $(m, n)$ are not nearest neighbors in $\mathscr{L}$. In this sense we say that this is a 1-dependent oriented percolation model.

We are going to show that when $\phi=0$, for any $\varepsilon>0$ there are $L$ and $T$ such that

$$
P((m, n) \text { is wet }) \geq 1-\varepsilon / 2 .
$$

By translation invariance we may consider the site $(0,0)$ in $\mathscr{L}$. Assume that at time 0 each site of the interval $I$ is occupied by one $(1,2)$. Let $r_{t}$ be the rightmost site occupied by $(1,2)$ and whose line of descent goes back to $I$. One of the ways $r_{t}=x$ moves to $x+1$ is when there is a creation of a 1 at $x+1$ followed by a creation of a 2 at $x+1$. Since $\phi=0, r_{t}$ only moves to the right so that

$$
r_{t} \geq N_{t}
$$

for all $t \geq 0$, where $N_{t}$ is a renewal process for which the mean time between two renewals is

$$
1 / \lambda_{1}+1 / \lambda_{2} \text {. }
$$

By the renewal theorem we know that almost surely

$$
\liminf _{t \rightarrow \infty} \frac{r_{t}}{t} \geq \frac{\lambda_{1} \lambda_{2}}{\lambda_{1}+\lambda_{2}}=\Lambda \text {. }
$$

Taking $L$ large enough and $T=5 L / 2 \Lambda$ we get that

$$
P\left(r_{T} \geq 3 L\right) \geq 1-\varepsilon / 4 .
$$


The leftmost site occupied by $(1,2)$ behaves in a symmetrical way and all the sites between the leftmost $(1,2)$ and the rightmost $(1,2)$ are occupied by $(1,2)$ 's. Thus,

$$
P((m, n) \text { is wet }) \geq 1-\varepsilon / 2 \text { if } \phi=0 .
$$

Observe that the above is true regardless of the appearance of 1's and 2's coming from the boundary of the box $B$. For fixed $L$ and $T$ there is $f>0$ such that, if $\phi<f$, then the probability of any occurrence of any of the Poisson processes with rate $\phi$ in the finite box $B$ is smaller than $\varepsilon / 2$. Thus,

$$
P((m, n) \text { is wet }) \geq 1-\varepsilon \text { if } \phi<f .
$$

At this point we have three different systems: the infinite predator-prey model, the predator-prey model restricted to the finite boxes $B_{m, n}$ and the oriented percolation model. Observe that the infinite predator-prey system will have at least as many sites occupied by $(1,2)$ 's as the predator-prey system restricted to the finite boxes $B_{m, n}$. This is so thanks to the free boundary conditions we have on each box. This proves that the predator-prey system dominates a 1-dependent oriented percolation. For $\varepsilon$ small enough it is known that there is percolation and that the density of wet sites is positive [see Durrett (1984)]. To get a stationary distribution for the predator-prey model, it is enough to start with all the sites of $\mathbf{Z}$ occupied by a $(1,2)$, then extract a convergent subsequence of the Cesaro averages. The limit is stationary since this process is Feller [see Property 1.8 in Chapter I in Liggett (1985)]. By comparison with oriented percolation, we see that the density of sites $(1,2)$ is strictly positive for this stationary distribution.

For the predator-prey model on $\mathbf{Z}^{d}(d \geq 2)$, we may embed the preceding 1-dimensional construction and get a stationary distribution in exactly the same way. The only difference is that the boundary of each $B_{m, n}$ is larger but since Poisson processes corresponding to $\phi$ do not occur in $B_{m, n}$, this has no influence on the construction. This completes the proof of Theorem 1.

3. Proof of Theorem 2. We will again make a comparison with oriented percolation. To avoid introducing oriented percolation in $\mathbf{Z}^{d+1}$ for $d \geq 2$, we will write the proof for $d=1$ and indicate how to proceed in higher dimensions.

Take $\mathscr{L}$ as in the proof of Theorem 2 and define the finite boxes

$$
A=[-2 L, 2 L] \times[0,2 T], \quad D=[-L, L] \times[T, 2 T],
$$

where $L$ and $T$ are integers to be chosen later. Define $\mathscr{C}$ to be the boundary of the box $A$ :

$$
\mathscr{C}=([-2 L, 2 L] \times\{0\}) \cup(\{-2 L\} \times[0,2 T]) \cup(\{2 L\} \times[0,2 T]) .
$$

We say that the site $(m, n)$ in $\mathscr{L}$ is wet if there are no 2's in the box $(m L, n T)+D$ whatever the state of the sites in $(m L, n T)+\mathscr{C}$ are. This defines a 1-dependent oriented percolation on $\mathscr{L}$.

Consider first the predator-prey model with $\phi=\infty$. For this model we have only the three states, $0,1,2$ : state $(1,2)$ is instantaneously transformed 
into state 2. So the 2's have birth rate $\lambda_{2}$ and death rate $\delta_{2}$. They evolve independently of the evolution of the 1's. Thus, the 2's are a subcritical contact process. We denote by $\xi_{t}^{y}$ a contact process on $\{0,2\}^{\mathbf{Z}}$ starting with a single 2 at $y$ at time 0 . If $\xi_{t}$ is a subcritical contact process we know by Bezuidenhout and Grimmett (1991) that there is a $c\left(\lambda_{2}, \delta_{2}\right)>0$ such that

$$
P\left(\xi_{t}^{y}(x)=2 \text { for some } t\right) \leq e^{-c\|x-y\|} .
$$

Define the survival time of the process $\xi_{t}^{y}$ by

$$
\tau^{y}=\inf \left\{t: \xi_{t}^{y}(x)=0 \text { for all } x \in \mathbf{Z}\right\} .
$$

Bezuidenhout and Grimmett (1991) have also proved that

$$
P\left(\tau^{0}>k\right) \leq e^{-c k} .
$$

Observe now that a 2 in the box $D$ must have originated somewhere in the boundary $\mathscr{C}$ of the box $A$. If the 2 comes from one of the two lateral sides of $A$, it means that a 2 has spread out at least $L$. If the 2 comes from the bottom of $A$, it means that a 2 has survived at least until $T$. So using the exponential decays, we get

$$
P(\text { there is a } 2 \text { in } D) \leq 2\left(2 T e^{-c L}\right)+(4 L+1) e^{-c T} .
$$

Thus, for any $\varepsilon>0$ we may pick $L=T$ large enough so that for $\phi=\infty$,

$$
P(\text { there is a } 2 \text { in } D) \leq \varepsilon / 2 .
$$

It is easy to see that as $\phi$ goes to infinity, the predator-prey model converges in distribution to the model with $\phi=\infty$ in the finite space-time box $A$. Thus, there is $F\left(\lambda_{2}, \delta_{2}\right)$ such that if $\phi>F$ then

$$
P(\text { there is a } 2 \text { in } D) \leq \varepsilon .
$$

So

$$
P((m, n) \text { is wet }) \geq 1-\varepsilon \text { for } \phi>F .
$$

We define $W_{n}$ to be the set of $m \in Z$ such that $(m, n)$ can be reached from $(0,0)$ by a path of wet sites that can only jump from $(j, k)$ to $(j-1, k+1)$ or to $(j+1, k+1)$. Let $l_{n}=\inf W_{n}$ and $r_{n}=\sup W_{n}$. Results by Durrett (1984) [see also Durrett and Neuhauser (1991)] imply that if $\varepsilon$ is small enough then the event $\left\{W_{n} \neq \varnothing, \forall n\right\}$ has positive probability (i.e., percolation occurs) and when this occurs we have

$$
\limsup _{n \rightarrow \infty} l_{n} / n \leq-a<0, \quad \liminf _{n \rightarrow \infty} r_{n} / n \geq a>0 .
$$

Suppose that $W_{n} \neq \varnothing$, let $(0,0),\left(i_{1}, 1\right), \ldots,\left(i_{n}, n\right)$ be the leftmost path from $(0,0)$ to $\left(l_{n}, n\right)$ and let $(0,0),\left(j_{1}, 1\right), \ldots,\left(j_{n}, n\right)$ be the rightmost path from $(0,0)$ to $\left(r_{n}, n\right)$. The definition of wet site implies that the regions $\left(i_{k} L, k T\right)+$ $D$ and $\left(j_{k} L, k T\right)+D$ do not contain any 2's and they overlap to form a connected region $\mathscr{V}$ which is vee-shaped. Observe that $\mathscr{V}^{c} \cap(R \times(0, n T))$ 
consists of two unbounded components (the outside) and one or more bounded components (the inside). In order to get a 2 in the inside of $\mathscr{V}^{c}$, a path of 2's would need to go through $\mathscr{V}$, which is impossible since 2's do not appear spontaneously but only by nearest-neighbor interaction. Therefore there can be no 2's in the inside of $\mathscr{V}^{c}$.

So when percolation occurs there is a linearly growing region for the predator-prey system in which there are no 2's. Percolation has positive probability to occur; if it does not occur, we wait until $W_{n}=\varnothing$ and then try again. Each trial is independent of what happened earlier, so eventually we will have a linearly growing region in which there are no 2's. Observe that all this is independent of the initial configuration. This completes the proof of Theorem 2 in dimension 1.

In $d>1$ we must compare the predator-prey system to a $(d+1)$ oriented percolation to show that if $\varepsilon>0$ is small, we get with positive probability a linearly growing cone that contains on 2's. Durrett (1992) proved the necessary percolation results. Observe also that the exponential decays for the contact process were proved in any dimension by Bezuidenhout and Grimmett (1991) and they can be used to prove that a renormalized site is wet with probability arbitrarily close to 1 . These are the two crucial ingredients to make the proof of Theorem 2 work in $d>1$.

\section{REFERENCES}

ANDJEL, E. and SchinaZI, R. (1996). A complete convergence theorem for an epidemic model. J. Appl. Probab. 33 741-748.

Bezuidenhout, C. and Grimmett, G. (1990). The critical contact process dies out. Ann. Probab. $181462-1482$.

Bezuidenhout, C. and Grimmett, G. (1991). Exponential decay for subcritical contact and percolation processes. Ann. Probab. 19 984-1009.

Bramson, M. and DurRetT, R. (1988). A simple proof of the stability criterion of Gray and Griffeath. Probab. Theory Related Fields 80 293-298.

Durrett, R. (1984). Oriented percolation in two dimensions. Ann. Probab. 12 99-1040.

DurRetT, R. (1988). Lecture Notes on Particle Systems and Percolation. Wadsworth, Belmont, CA.

DuRRett, R. (1991). The contact process, 1974-1989. Lectures in Appl. Math. 27. Amer. Math. Soc., Providence, RI.

DuRRETt, R. (1992). Multicolor particle systems with large threshold and range. J. Theoret. Probab. 5 127-152.

DurRetT, R. (1993). Predator-prey systems. In Asymptotic Problems in Probability Theory: Stochastic Models and Diffusions on Fractals (K. D. Elworthy and N. Ikeda, eds.) 37-58. Longman, Essex, England.

DurRett, R. (1995). Ten Lectures on Particle Systems. Lecture Notes in Math. 1608. Springer, Berlin.

Durrett, R. and Neuhauser, C. (1991). Epidemics with recovery in $D=2$. Ann. Appl. Probab. 1 189-206.

DurRett, R. and Schinazi, R. (1993). Asymptotic critical value for a competition model. Ann. Appl. Probab. 3 1047-1066.

HARRIS, T. (1972). Nearest neighbor Markov interaction processes on multidimensional lattices. Adv. in Math. 9 66-89.

Isham, V. (1995). Stochastic model of host-macroparasite interaction. Ann. Appl. Probab. 5 $720-740$. 
Kang, H., Krone, S. and Neuhauser, C. (1995). Stepping stone models with extinction and recolonization. Ann. Appl. Probab. 5 1025-1060.

Liggett, T. (1985). Interacting Particle Systems. Springer, New York.

Sato, K., Matsuda, H. and Sasaki, A. (1994). Pathogen invasion and host extinction in lattice structured populations. J. Math. Biol. 32 251-268.

SchinAZI, R. (1996). On an interacting particle system modeling an epidemic. J. Math. Biol. 34 915-925.

Department of Mathematics

UNIVERSITY OF COLORADO

Colorado Springs, Colorado 80933-7150

E-MAIL: schinazi@vision.uccs.edu 